\title{
Simultaneous bilateral Anterior Cruciate Ligament (ACL) reconstruction with use of hamstring tendon autografts: a case report
}

\author{
Matjaz Sajovic \\ Department for Orthopedics and Sports Trauma Surgery, General Teaching Hospital, Celje, Slovenia
}

Email addres:

matjaz.sajovic@triera.net (M. Sajovic)

\section{To cite this article:}

Matjaz Sajovic. Simultaneous Bilateral Anterior Cruciate Ligament (ACL) Reconstruction with use of Hamstring Tendon Autografts: a Case Report, Journal of Surgery. Vol. 1, No. 1, 2013, pp. 1-5. doi: 10.11648/j.js.20130101.11

\begin{abstract}
Among patients presenting with ACL deficient knees, the incidence of bilaterality is reported to be between $2 \%$ and $4 \% .{ }^{(1)}$ We have presented an unusual problem of a patient with chronic bilateral ACL-deficient knees and constitutionally very thin patellar tendons. Author decided to perform one-stage bilateral ACL reconstructions using hamstring tendon autografts so as not to weaken his quadriceps muscles by compromising his extensor mechanism. At 7 years follow-up, the patient's opinion was that both ACL reconstructed knees had normal function and he was still on his preinjury level of activity. Two-stage bilateral ACL reconstruction is much more time consuming for the patient and expensive for health insurance, so in such cases, we recommend performing one-stage, bilateral ACL reconstructions using hamstring tendon autografts as a method that is effective and reproducible for a timely return of motion, strength, and function.
\end{abstract}

Keywords: ACL Rupture; One-Stage Bilateral ACL Reconstruction; Rehabilitation

\section{Introduction}

The anterior cruciate ligament (ACL) rupture is the most common serious injury of the knee. Enhancement of participants of all ages in sports resulted in increasing of the incidence of the ACL tears. The goals of ACL reconstructions are to decrease symptoms, improve function, and return patients to their preinjury level of activity. ACL reconstruction is a common procedure that usually allows predictable and timely return to function for the patient. A variety of graft sources, such as autografts, allografts, and synthetic grafts, have been used for ACL reconstructions. Presently, the autologous patellar tendon $\left({ }^{12,14,21,26}\right)$ or doubled semitendinosus and gracilis tendons $(2,13,23,29)$ are the most frequently used grafts for intraarticular ACL reconstruction. Disadvantages of patellar tendon autografts are risk of patellar fracture, potential increase in patellofemoral pain, and retained patellar tendon weakness or rupture. $\left({ }^{20,4,19}\right)$ Disadvantages of hamstring tendon autografts include potential hamstring muscle weakness $\left({ }^{6}\right)$ and slower healing of the graft attachment site. $\left({ }^{30}\right)$ Graft fixation is crucial in ACL reconstruction and is the weakest link in the initial 6 to 12 weeks period, during which healing of the graft to the host bone occurs. Aperture fixation by interfe- rence screw either patellar tendon or four strand hamstring tendon autograft, provides required tensile load of construct for accelerated postoperative rehabilitation. $\left({ }^{25}\right)$

Bilateral ACL rupture is not common clinical problem, but the incidence of the bilateral knee injuries increasing especially among highly sports active population. Within patients with ACL-deficient knees, the incidence of bilaterality is reported to be between $2 \%$ and $4 \%$. $\left({ }^{1,27}\right)$ Mechanism of injury rarely causes simultaneous bilateral ACL tear. Usually, unilateral injury of the knee has occurred and later on one during further sports activity contralateral knee injury has taken place. Several risk factors for tearing the ACL have been evaluated in the literature. Inadequate or compromised conditioning, experience, muscle recruitment patterns, $\left({ }^{8}\right)$ and proprioception $\left({ }^{22}\right)$ have been described as partially controllable, trainable characteristics. ( $\left.{ }^{16}\right)$ It has also been suggested that anatomical particularity of narrow intercondylar notch width index and an increased tibial slope increase the risk of ACL injury. However, Lombardo et al. $\left({ }^{18}\right)$ reported that notch width index did not predict the rate of ACL injury. Familial predisposition could be associated factor for the ACL tear. ( ${ }^{7}$ )

Present paper describes a successful surgical treatment of the case of chronic bilateral ACL deficiency with episodes of instability in both knees supplied with brief litera- 
ture review and discussion.

\section{Case Report}

A 21 years old man was evaluated at our clinic who reported bilateral knee problems, with the left knee worse than the right. His history of injury actually went back two years, at which time his first injury occurred while he was skiing. Clinically, it appeared as though he had sustained bilateral ACL tear and was treated in some other hospital with bilateral cast immobilization for 5 weeks followed by rehabilitation. His level of activity had been decreased and he did not have for two years a lot of problems with instability of the knees. Then, while exercising martial arts, his left knee locked. On examination, he had a mild effusion in the left knee and no effusion of the right knee. There was no marked quadriceps atrophy on either leg. His range of movement in right knee was full from $5^{\circ}$ of hyperextension to $135^{\circ}$ of flexion. His left knee was locked in $25^{\circ}$ of flexion. He had $2+$ Lachman sign in both knees and $2+$ pivot shift sign in right knee. Pivot shift test examination of the left locked knee was impossible. Clinically, in addition to his bilateral chronic ACL tears, he had a bucket-handle tear of the medial meniscus in the left knee, which was responsible for locked knee. Preoperative Lysholm knee score for left knee was 22 and for right knee was 43. Absolute KT2000 anterior translation at $134 \mathrm{~N}$ was $11.6 \mathrm{~mm}$ in left knee and $12.4 \mathrm{~mm}$ in right knee.

The treatment options were discussed with patient, who was willing to conduct healthy sportive lifestyle. Taking into account patient's age and his wishes one-stage bilateral (simultaneous) ACL reconstructions using hamstring tendon autografts were chosen as an optimal treatment for this case. The main reason to perform one stage bilateral ACL procedure was also the fact that staged bilateral procedures are much more time consuming for the patient.

On the day of the surgery, the arthroscopic procedure has been performed on his left knee first. The operating room set-up included the use of two exclusion arthroscopy drapes, one-camera stack system and a single set of reconstruction instruments to allow one-stage bilateral surgery by one surgical team. Both knees were prepared and draped separately. During arthroscopic procedure of left knee, we found a displaced bucket-handle tear of the medial meniscus, that was repaired and degenerative lateral meniscus parrot beak tear, which was excised. After ACL reconstruction, the tourniquet was released and the knee was wrapped with an elastic bandage. Then, an arthroscopic ACL reconstruction was performed on the other leg.

The patient began with the rehabilitation process the day after the surgery. He did continuous passive motion for both knees and active flexion/extension exercises. We performed electrostimulation of the musculus quadriceps femoris and cryotherapy of the knee. At the same time he attempted to walk with the help of walking frame. He did not use knee braces in rehabilitation process. The patient achieved full active extension and 90 degree flexion of both knees on the third day after surgery, which was also the date of his discharge. He was able to walk without chrutches due to sufficient muscle control. The patient continued the second phase of rehabilitation in local physical therapy infirmary. The aim of the process during next five weeks was to achieve complete active flextion. Emphasis was on achieving functional gate. He began with basic exercises of proprioception (standing on one's toes or heels and shifting weight forwards, backwards, left and right). He was taught muscle-building exercises with various aids (elastic bands, weights ) in the sitting and lying position as well as appropriate stretching techniques for the thigh and calf mucles. We started with the use of an exercise bicycle the third post operative week. From the fifth week onwards, the patient continued with close kinetic exercises. He gained in muscular endurance and vital capacity. He worked on increasing muscle mass in the gym, where he trained all muscle groups of the lower extremities along with isokinetic training. He gradually began developing explosivness of the legs muscle. He did more advanced proprioception exercises coupled with elements of coordination (Figure 1). 4 months after the surgery, he began with running and jumping squats. Full sports activities were recommended after seven months.

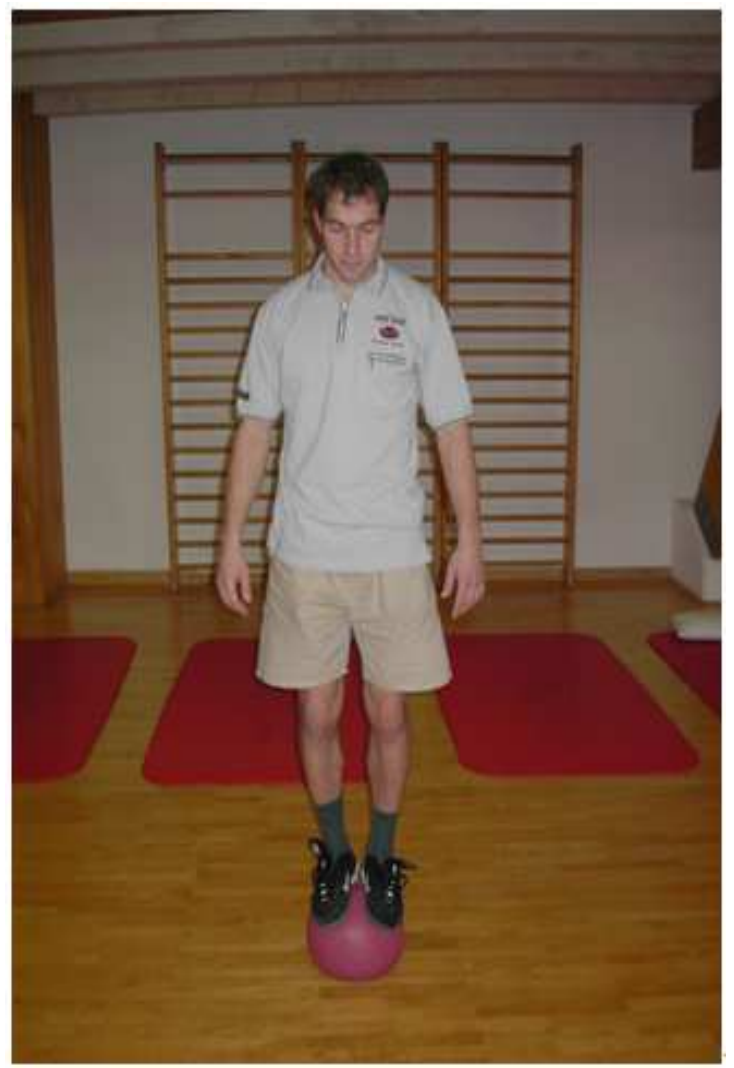

Figure 1. Proprioception and coordination exercise six weeks postoperatively.

At 7 years follow-up, the patient's opinion was that both ACL reconstructed knees had normal function and he was still on his preinjury level of activity. The overall result of the Lysholm knee score for left knee was 95 and for right 
knee was 100 . On clinical examination, he had no effusion and his range of movement was full from $5^{\circ}$ of hyperextension to $135^{\circ}$ of flexion in both knees. He had negative Lachman and pivot shift signs in both knees. Absolute KT2000 anterior translation at $134 \mathrm{~N}$ was $4.0 \mathrm{~mm}$ in left knee and $4.1 \mathrm{~mm}$ in right knee. The radiographs taken at followup did not show joint space narrowing or presence of osteophytes. At follow-up we performed the Cybex II isokinetic testing of 5 cycles at a velocity of $60 \%$ s (Figure 2 ). Comparing both sides, we found less than $10 \%$ difference between the legs. Overall IKDC score grade A was obtained in both ACL reconstructed knees.

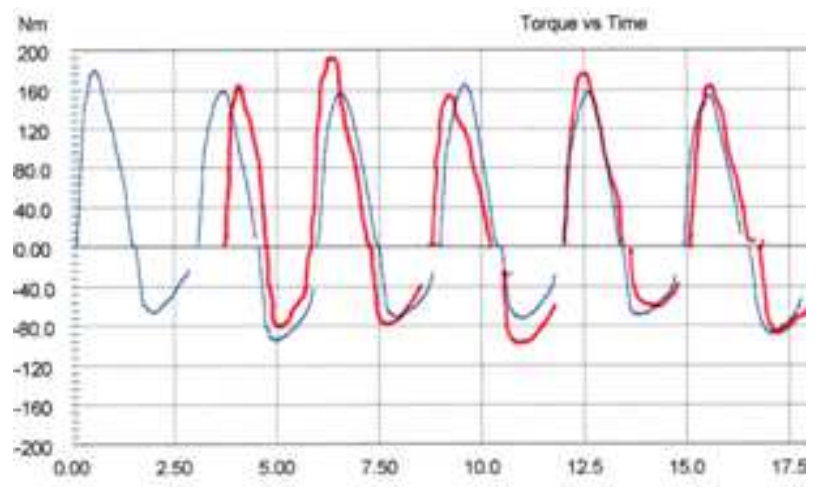

Figure 2. Cybex II isokinetic testing of 5 cycles at a velocity of $60 \% \mathrm{~s}$

\section{Discussion}

In this case report an unusual case of chronic bilateral ACL-deficient knees and constitutionally very thin patellar tendons was described and we recommend, in such cases, performing one-stage, bilateral ACL reconstructions using hamstring tendon autografts as an optimal method that is effective and reproducible for a timely return of motion, strength, and function. The simultaneous bilateral ACL reconstruction is clinical and cost effective treatment for patients presenting symptomatic bilateral ACL deficiency. Staged bilateral procedures are much more time consuming for the patient, and it requires two operative procedures as well as two rehabilitation programs.

Despite the otherwise abundant literature regarding ACL reconstruction, there is very little information regarding strategies for patients with bilateral ACL deficient knees. The incidence of bilaterality for ACL ruptures is reported to be between $2 \%$ and $4 \%$. $\left({ }^{1,27}\right)$ In spite of reports on simultaneous bilateral ACL ruptures in the literature, the vast majority of patients presenting with bilateral ACL deficient knees sustain non-simultaneous bilateral ruptures. A combination of many risk factors for tearing the ACL (e.g., intercondylar notch stenosis, increased tibial slope, hormonal influences, joint laxity, muscle strength, proprioception, limb alignment, experience, conditioning) have been suggested in the literature.

To our knowledge Jari and Shelbourne $\left({ }^{11}\right)$ were the first to publish results concerning the bilateral ACL reconstruction as a single procedure. They have performed the simul- taneous bilateral ACL reconstruction using the patellar tendon autograft. Their results showed, that in the short term, there was no statistically significant difference in postoperative results between patients who had simultaneous bilateral ACL reconstructions and patients who had unilateral ACL reconstruction. These results indicate that there might be an advantage in performing simultaneous ACL reconstructions to stage procedures, which would involve two operations and rehabilitation programs. In the Larson et al. study, the majority of patients $(80 \%)$ underwent patellar tendon allograft ACL reconstruction. $\left({ }^{15}\right)$ They performed a more comprehensive analysis of costs incurred both on the day of surgery and during rehabilitation. They found that performing bilateral ACL reconstruction at a single setting resulted in a total cost savings of more than $\$ 3.750$ per knee. They found that day-of-surgery charges made up the majority of savings, postoperative rehabilitation accounted for nearly $15 \%$ of the total. Economic aspects play a significant role in healthcare delivery. In addition to lower costs, only one period off work and one period of rehabilitation are needed, which results in less disruption to the lives of the patients and caregivers.

Present paper illustrates a problem that arises when the patient cannot cope with bilateral ACL-deficient knees. Some patients with this condition decrease their activities so much, that quadriceps weakness persists despite regular rehabilitation exercises. In this particular case, author used doubled semitendinosus and gracilis tendon autografts in one-stage bilateral ACL reconstruction in order to avoid further postoperative quadriceps muscles weakness and reducing possibility of postoperative complication. Preoperatively, the patient had constitutionally very thin patellar tendons so harvesting the bone-patellar tendon-bone transplants could seriously compromise his extensor mechanism. By using hamstring tendon autografts, we allowed immediately after surgery aggressive strengthening of the quadriceps muscle of both legs. At the same time, the rehabilitation program was focused immediately on achieving functional gate and indipendence of the patient during dayto-day activities.

Another option would have been the use of a patellar tendon allograft, which would eliminate any donor site problems. $(9,17,28)$ However, an allograft does have potential problems, including availability, storage, sterilisation, and disease transmission. ${ }^{3,5,24}$ ) The allograft has also been shown to have physiologic differences from autografts, with slower revascularization and recollagenization and suboptimal healing, suggested by immunogenic studies. $\left({ }^{30}\right)$ The use of an aggressive rehabilitation program may not be desirable with an allograft, $\left({ }^{10}\right)$ and we believe postoperative stability is certainly not as reproducible as that obtained with using autograft.

If the synthetic ligaments were used to solve the problem, it would probably result in a worst case scenario. Synthetic ligaments have a higher complication rate than allograft and autograft reconstructions. They have been associated with a high rate of synovitis. An in vitro study in pigs 
found that ingrowth into the scaffold does not occur. $\left({ }^{31}\right)$ Also, the carbon fibers do not adhere to the bony channels, and the peak tensile strength of the ligaments after reconstruction with a carbon scaffold was one third the strength of a normal ACL at 4 months postoperatively. Between 1987 and 1989 we have performed at our clinic 12 ACL reconstructions using the prosthetic implant Gore-Tex. In next 6 years we had to make revision procedures in 9 patients because of recurrent instability, chronic effusions, and synovitis.

Author presented an unusual problem of a patient with chronic bilateral ACL-deficient knees and constitutionally very thin patellar tendons. We recommend, in such cases, performing one-stage, bilateral ACL reconstructions using hamstring tendon autografts as a method that is effective and reproducible for a timely return of motion, strength, and function.

\section{References}

[1] Anderson AF, Lipscomb AB, Liudah KJ, et al. Analysis of the intercondylar notch by computed tomography. Am J Sports Med 1987; 15: 547-552.

[2] Anderson AF, Snyder RB, Lipscomb AB Sr. Anterior cruciate ligament reconstruction using the semitendinosus and gracilis tendons augmented by the Losee iliotibial band tenodesis: A long-term study. Am J Sports Med 1994; 22: 620-626.

[3] Asselmeier MA, Caspari RB, Bottenfield S. A review of allograft processing and sterilization techniques and their role in transmission of the human immunodeficiency virus. Am J Sports Med 1993; 21: 170-175.

[4] Bonamo JJ, Krinick RM, Sporn AA. Rupture of the patellar ligament after use of its central third for anterior cruciate reconstruction. A report of two cases. J Bone Joint Surg 1984;66A:1294-1297.

[5] Buck BE, Resnick L, Shah SM, et al. Human immunodeficiency virus cultured from bone: Implications for transplantation. Clin Orthop 1990; 251: 249-253.

[6] Coombs R, Cochrane T. Knee flexor strength following anterior cruciate ligament reconstruction with the semitendinosus and gracilis tendons. Int J Sports Med 2001; 22: 618-622.

[7] Flynn RK, Pedersen CL, Birmingham TB, et al. The familial predisposition toward tearing the anterior cruciate ligament. A case control study. Am J Sports Med 2005; 33:2328.

[8] Harmon KG, Ireland ML. Gender differences in noncontact anterior cruciate ligament injuries. Clin Sports Med. 2000; 19: 287-302.

[9] Harner CD, Olson E, Irrgang JJ, et al. Allograft versus autograft anterior cruciate ligament reconstruction. 3- to 5year outcome. Clin Orthop 1996; 324: 134-144.

[10] Jackson DW, Grood ES, Goldstein JD, et al. A comparison of patellar tendon autograft and allograft used for anterior cruciate ligament reconstruction in the goat model. Am $\mathbf{J}$
Sports Med 1993; 21: 176-185.

[11] Jari S, Shelbourne KD. Simultaneous bilateral anterior cruciate ligament reconstruction. Am J Sports Med 2002; 30: 891-895.

[12] Jones KG. Reconstruction of the anterior cruciate ligament. A technique using the central one-third of the patellar ligament. J Bone Joint Surg 1963; 45A: 925-932.

[13] Karlson JA, Steiner ME, Brown CH, et al. Anterior cruciate ligament reconstruction using gracilis and semitendinosus tendons. Comparison of through-the-condyle and over-thetop graft placements. Am J Sports Med 1994; 22: 659-666.

[14] Lambert KL. Vascularized patellar tendon graft with rigid internal fixation for anterior cruciate ligament insufficiency. Clin Orthop 1983; 172: 85-89.

[15] Larson CM, Fischer DA, Smith JP, et al. Bilateral anterior cruciate ligament reconstruction as a single procedure. Evaluation of cost and early functional results. Am J Sports Med 2004; 32: 197-200.

[16] Lephart SM, Ferris CM, Fu FH. Risk factors associated with noncontact anterior cruciate ligament injuries in female athletes. Instr Course Lect. 2002; 51: 307-310.

[17] Lephart SM, Kocher MS, Harner CD, et al. Quadriceps strength and functional capacity after anterior cruciate ligament reconstruction. Patellar tendon autograft versus allograft. Am J Sports Med 1993; 21: 738-743.

[18] Lombardo S, Sethi PM, Starky C. Intercondylar notch stenosis is not risk factor for anterior cruciate ligament tears in professional male basketball players. An 11-year prospective study. Am J Sports Med 2005; 33: 29-34.

[19] Marumoto JM, Mitsunaga MM, Richardson AB, et al. Late patellar tendon ruptures after removal of the central third for anterior cruciate ligament reconstruction: A report of two cases. Am J Sports Med 1996; 24: 698-701.

[20] Mastrokalos DS, Springer J, Siebold R, et al. Donor site morbidity and return to the preinjury activity level after anterior cruciate ligament reconstruction using ipsilateral and contralateral patellar tendon autograft. Am J Sports Med 2005;33: 85-93.

[21] O'Brien SJ, Warren RF, Pavlov H, et al. Reconstruction of the chronically insufficient anterior cruciate ligament with the central third of the patellar ligament. J Bone Joint Surg 1991; 73A: 278-286.

[22] Ochard J, Seward H, McGiven J, et al. Intrinsic and extrinsic risk factors for anterior cruciate ligament injury in Australian footballers. Am J Sports Med. 2001;29: 196-200.

[23] Pinczewski LA, Deehan DJ, Salmon LJ, et al. A five-year comparison of patellar tendon versus four-strand hamstring tendon autograft for arthroscopic reconstruction of the anterior cruciate ligament. Am J Sports Med. 2002; 30: 523-536.

[24] Roberts TS, Drez D Jr, McCarthy W, et al. Anterior cruciate ligament reconstruction using freeze-dried, ethylene oxide sterilized bone-patellar tendon-bone allografts. Two year results in 36 patients. Am J Sports Med 1991; 19: 35-41.

[25] Rowden NJ, Sher D, Rogers GJ, et al. Anterior cruciate ligament graft fixation: Initial comparison of patellar tendon and semitendinosus autografts in young fresh cadavers. Am 
J Sports Med 1997;25: 472-478.

[26] Shelbourne KD, Gray T. Anterior cruciate ligament reconstruction with autogenous patellar tendon graft followed by accelerated rehabilitation. A two- to nine-year followup. Am J Sports Med 1997; 25: 786-795

[27] Souryal TO, Moore HA, Evans JP. Bilaterality in anterior cruciate ligament injuries: Associated intercondylar notch stenosis. Am J Sports Med 1988; 16(5): 449-454.

[28] Stringham DR, Pelmas CJ, Burks RT, et al. Comparison of anterior cruciate ligament reconstruction using patellar tendon autograft or allograft. Arthroscopy 1996; 12: 414-421.
[29] Wagner M, Kääb MJ, Schallock J, Haas NP, Weiler A. Hamstring tendon versus patellar tendon anterior cruciate ligament reconstruction using biodegradable interference fit fixation: A prospective Matched-Group Analysis. Am J Sports Med. 2005;33:1327-1336.

[30] West RV, Harner CD. Graft selection in anterior cruciate ligament reconstruction. J Am Acad Orthop Surg 2005; 13: 197-207.

[31] Zoltan DJ, Reinecke C, Indelicato PA. Sythetic and allograft anterior cruciate ligament reconstruction. Clin Sports Med 1988; 7: 773-784. 